\title{
Directional coupling and branching of optical signals by dark beams
}

\author{
S. Chervenkov ${ }^{1}$, D. Neshev ${ }^{1}$, G. G. Paulus ${ }^{2}$, A. Dreischuh ${ }^{1}$ and H. Walther ${ }^{2,3}$ \\ ${ }^{1}$ Sofia University, Department of Quantum Electronics, 5, J. Bourchier Blvd., BG-1164, Bulgaria \\ ${ }^{2}$ Max-Plank-Institut für Quantenoptik, Hans-Kopfermann-Str. 1, D-85748 Garching, Germany \\ ${ }^{3}$ Ludwig-Maximillians-Universität, Sektion Physik, Am Coulombwall 1, D-85747 Garching, Germany
}

\begin{abstract}
Various types of dark optical beams with respect to their phase dislocation are discussed and their propagation dynamics is analyzed. On the basis of numerical results $1 \mathrm{D}$ and 2D optical couplers are proposed. The respective energy efficiencies for each of the output channels are estimated. Multiple charged optical vortices and their topological instability are presented. Through a proper type of perturbation applied to these vortices they could be split into several sub-beams, thus giving the opportunity of proposing all-optical branching schemes. Two output channel distribution schemes for such an optical brancher are discussed.
\end{abstract}

Keywords: non-linear optics, dark beam, phase dislocation, perturbation, coupler, brancher

\section{INTRODUCTION}

The phenomenon of optical beam redirection to a certain output position is referred to as directional coupling. On the other hand single beam splitting into several sub-beams is called branching in space.

The propagation of a dark beam in a self-defocusing nonlinear medium leads to refractive index change in a way to induce an effective optical waveguide. This property could be successfully used for guiding weak (not destroying the nonlinear waveguide) signal beams and pulses. The ability to manipulate the propagation dynamics of dark beams gives an opportunity to control the output position of the guided signals or to split them in space. Due to this reason dark beams could be used in all-optical directional coupling and branching schemes.

Two possible approaches for realizing these schemes are investigated. In the first one changes of the type of the phase dislocation (screw, mixed edge-screw (ES), or step-screw (SS)) are implemented. The second one is based on the topological instability of multiple-charged optical vortices (OVs) under the action of perturbation.

\section{DARK BEAMS AND OPTICAL SOLITONS}

Solitons can be understood as a balance between the effect of dispersion and that of nonlinearity ${ }^{1}$. The evolution of electric field amplitude in a cubic non-linear medium is described by the generalized non-linear Schrödinger equation (GNLSE). It has the following form:

$$
-i \frac{\partial E}{\partial z}+\alpha\left(\frac{\partial}{\partial x^{2}}+\frac{\partial}{\partial y^{2}}\right) E+\beta_{2} \frac{\partial E}{\partial t^{2}}+\gamma f\left(|E|^{2}\right) E=0,
$$

where the variables $x, y$ and $t$ stand for the transverse spatial and the temporal coordinates, respectively, $z$ is the propagation coordinate, and $E$ designates the electric field amplitude. The non-linearity of the Kerr and Kerr-like medium is manifested through the intensity dependent refractive index change. Generally, the non-linear refractive index can be presented by the relation

$$
n^{2}\left(x, y,|E|^{2}\right)=n_{0}^{2}(x, y)+f\left(|E|^{2}\right),
$$

where the non-linear refractive index correction $f\left(|E|^{2}\right)$ could depend on the intensity in various ways:

$$
f\left(|E|^{2}\right)= \begin{cases}\alpha_{1}|E|^{2} & \text { (Kerr medium) } \\ \frac{n_{0} n_{2}|E|^{2}}{1+\gamma^{2}|E|^{2} n_{2} / n_{0}} & \text { (two-level saturable medium) } \\ \alpha_{1}|E|^{2}-\alpha_{2}|E|^{2} & \text { (competitive cubic medium) }\end{cases}
$$


The exact one-dimensional and the only known dark two-dimensional solutions of the GNLSE comprise a specific class of self-supported waves called solitons. Solitons could be bright and dark. The shape of the bright ones is described by the formula $U(z, t)=a \operatorname{sech}(a t) e^{i a 2 z}$, the phase distribution is constant (Fig. 1). The envelope of dark solitons ${ }^{2}$ is described mathematically by the distribution $U(z, t)=U_{0} \tanh \left(U_{0} t\right) e^{i 2 U_{0}^{z}}$. According to their phase profile dark solitons are subdivided into two types: black (Fig. 2) and gray (Fig. 3) ones. In the case of black solitons $\pi$-phase jump is present, phase is not defined and, hence, at this point intensity is zero. In the case of gray solitons phase changes gradually, no phase jump is present, and that's why the intensity dip does not reach the zero level.

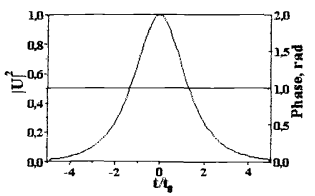

Fig. 1 Bright soliton

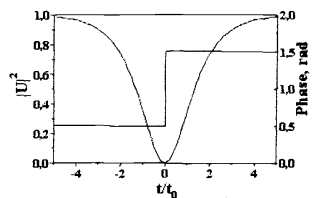

Fig. 2 "Black" soliton

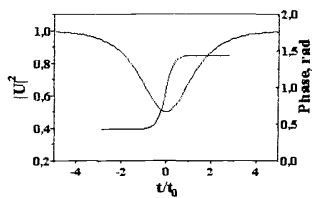

Fig. 3 "Gray" soliton

In the present work dark beams will be investigated. These beams are not solitons since dark beams with SS or ES mixed phase dislocation have non-zero transverse velocity. Further they will be referred to as odd dark beams (ODB). The multiple-charged optical vortices are not stable under perturbations and decay into single-charged ones.

\section{MIXED PHASE DISLOCATIONS}

Depending on the phase dislocation nested in the background beam, the dark beam could be redirected to a certain output position, thus giving the opportunity of producing an optical coupler. Two possible types of mixed phase dislocations ${ }^{3,4}$ are exploited: step-screw (SS) type and edge-screw (ES) type. The SS type of phase dislocation has transverse profile described by

$$
\Phi_{\alpha, \beta}^{S S}(x, y)=\Delta \Phi\left\{\frac{1-\alpha}{2} \operatorname{sgn}(y)-\frac{\beta}{\pi} \arctan \left(\frac{\alpha y}{x+b \beta}\right)\right\}, \quad \alpha=\left\{\begin{array}{ll}
0 & \text { for }|x| \leq b \\
1 \text { and } \beta=-1 & \text { for } x>b \\
1 \text { and } \beta=1 & \text { for } x \leq-b
\end{array} .\right.
$$

The edge-screw (ES) type is described by the expression, giving the dependency of phase on the transverse coordinates

$$
\Phi^{E S}(x, y)=\frac{\Delta \Phi}{2 \pi}\left\{\arctan \left(\frac{y}{x+b}\right)-\arctan \left(\frac{y}{x-b}\right)\right\} .
$$

In Eqs. 4 and $5 b$ stands for the length of the dislocation.

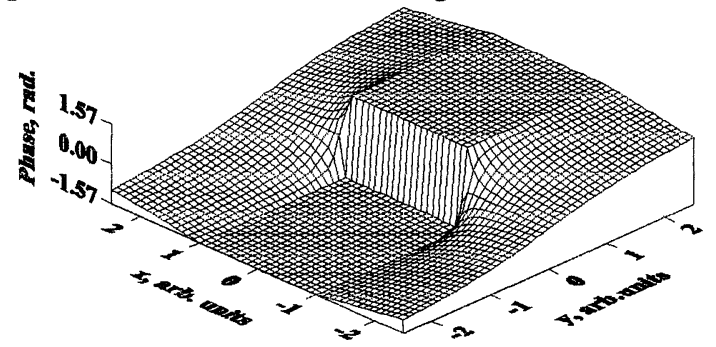

Fig. 4. Step-screw (SS) type of phase dislocation.

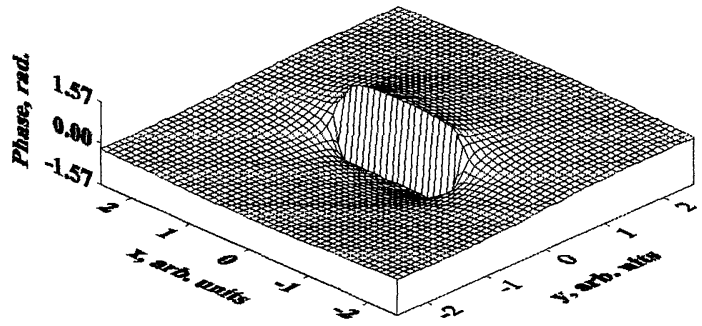

Fig. 5. Edge-screw (ES) type of phase dislocation.

\section{NUMERICAL MODEL AND RESULTS}

In the present work a simulation of dark beam propagation in Kerr-like non-linear medium is carried out. As a basis for the computer simulation a system of GNLSEs for the dark (D) and bright signal (B) beams is exploited:

$$
i \frac{\partial E_{D}}{\partial \zeta}+\frac{1}{2} \Delta_{\perp} E_{D}-\frac{L_{D i f f}}{L_{N L}} \frac{\left|E_{D}\right|^{2} E_{D}}{\left(1+s\left|E_{D}\right|^{2}\right)^{\gamma}}=0
$$




$$
i \frac{\partial E_{B}}{\partial \zeta}+\frac{1}{2} \frac{\lambda_{B}}{\lambda_{D}} \Delta_{\perp} E_{B}-\sigma \frac{L_{D i f f}}{L_{N L}} \frac{\left|E_{D}\right|^{2} E_{B}}{\left(1+s\left|E_{D}\right|^{2}\right)^{\gamma}}=0 .
$$

In the model it is assumed that the intensity-dependent refractive index correction is expressed by

$$
\Delta n=n_{2}\left|E_{D}\right|^{2} /(1+s)^{\gamma}
$$

The results obtained by the numerical simulation show that the transverse velocity of the dark beam with ES phase dislocation is greater than the velocity of the beam with SS phase profile. The quantity $\mathrm{I}^{\mathrm{ID}}$ DSS designates the reference intensity corresponding to that required to support ID DSS.

Because of the different propagation dynamics (at the same initial conditions) the ES and SS phase dislocations could be successfully used for addressing different output channels and thus for producing an all-optical coupler. On the basis of the properties discussed two schemes for 1D and 2D couplers are proposed.

\section{COUPLING SCHEMES}

\subsection{One dimensional (1D) coupler}

The scheme proposed consists of five output channels, each one aligned to a photodetector. The data show that each of the dark beams can be forced to address only a certain channel. The coupling energy efficiency to each of the channels is different, being the greatest for the zero-th channel. The disposition of the output channels in the 1D coupler is presented in Fig. 6. All obtained results are summarized in Table 1.

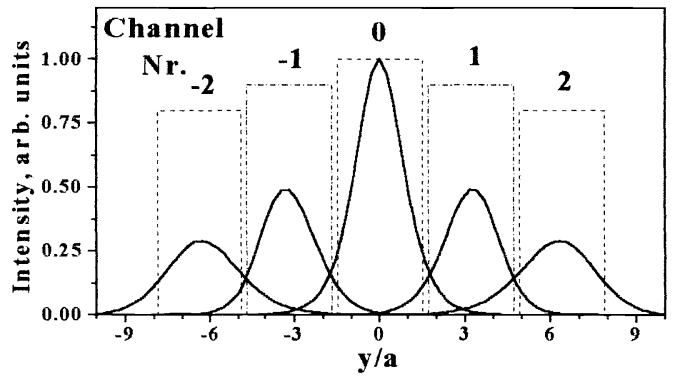

Fig. 6 Positions and shapes of the signal beams at $\zeta=10$. Channel 0 is assumed to be supported by an OVS, channels 1 and -1 by ODBs with SS phase dislocations $\left(\mathrm{b} / \mathrm{a}=1.5\right.$; both at $\left.\mathrm{I}=1.5 \mathrm{I}^{1 \mathrm{D}} \mathrm{DSs}\right)$. Channels 2 and -2 are addressed by ODBs with ES phase dislocations $\left(\mathrm{b} / \mathrm{a}=2.5\right.$ and $\left.\mathrm{I}=1.25 \mathrm{I}^{1 \mathrm{D}} \mathrm{DSS}\right)$.

\begin{tabular}{|c|c|c||c|c|c|}
\hline \multirow{2}{*}{$\begin{array}{c}\text { Guiding } \\
\text { beam }\end{array}$} & \multicolumn{5}{|c|}{ Efficiency in coupling to channel } \\
\cline { 2 - 6 } & -2 & -1 & 0 & 1 & 2 \\
\hline OVS & $0.3 \%$ & $9.5 \%$ & $74.7 \%$ & $9.5 \%$ & $0.3 \%$ \\
\hline SS ODB & $0.5 \%$ & $0.2 \%$ & $9.6 \%$ & $70.7 \%$ & $5.6 \%$ \\
\hline ES ODB & $56 \%$ & $14.1 \%$ & $1.4 \%$ & $1.6 \%$ & $0.8 \%$ \\
\hline
\end{tabular}

Table 1. Energy efficiencies for each of the output channels.

\subsection{Two-dimensional (2D) coupler}

The 2D coupler exploits the same principle as the one-dimensional one. The output channels are arranged in the form of a $3 \times 3$ matrix. Table 2 contains information about the type of the ODB addressing the respective channel and about the energy efficiencies in coupling the signal to some of the channels. As seen, the highest efficiencies are achieved for channels $\mathrm{C}_{11}$, $\mathrm{C}_{22}$ and $\mathrm{C}_{23}$, addressed by ES ODB, OVS, and SS ODB, respectively. The simulations show that the energy losses are different for the different types of dark beams, being the least for OVSs. The cross-section of the output energy distribution is depicted in Fig. 7. 


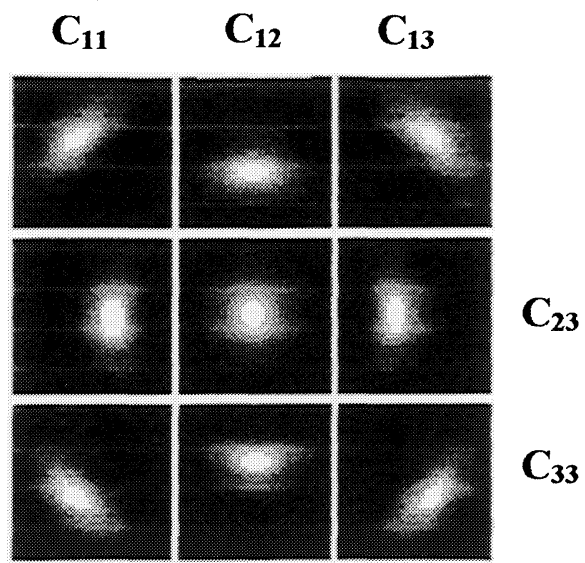

Fig. 7 The data refer to $\zeta=10, \mathrm{I}=1.5 \mathrm{I}^{1 \mathrm{D}}$ DSs, and channel width/height 1.5 times the input signal FWHM. The ratio $\mathrm{b} / \mathrm{a}=1.5$ in the SS case and 3.4 in the ES case, and $\Delta \Phi=\pi$.

\begin{tabular}{|c|c|c|c|c|c|c|}
\hline \multirow[b]{2}{*}{$\begin{array}{c}\text { Guiding } \\
\text { beam }\end{array}$} & \multicolumn{6}{|c|}{ Efficiency in coupling to channel } \\
\hline & $C_{11}$ & $C_{12}$ & $C_{13}$ & $\mathbf{C}_{21}$ & $\mathbf{C}_{22}$ & $\mathrm{C}_{23}$ \\
\hline OVS & 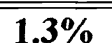 & $7.3 \%$ & $1.3 \%$ & $7.3 \%$ & $61.6 \%$ & $7.3 \%$ \\
\hline SS ODB & $\mathbf{0 . 2 \%}$ & $2.4 \%$ & $12.6 \%$ & $0.1 \%$ & $6.1 \%$ & $49.6 \%$ \\
\hline ES ODB & $47.1 \%$ & $5.5 \%$ & $0.7 \%$ & $5.5 \%$ & $\mathbf{0 . 3 \%}$ & $0.5 \%$ \\
\hline
\end{tabular}

Table 2. Energy efficiencies per each of the output channels

\section{BRANCHING BY DECAYING OF OPTICAL VORTICES}

The process of branching ${ }^{5,6}$ could be realized by using the topological instability of multiple charged optical vortices ${ }^{7,8}$ (OVs). When applying a small perturbation to an OV the latter could be split. So using this property of OVs by inducing a proper kind of perturbation to the guiding OV the splitting process could be controlled. In this way the number of the output channels and their relative energy can be varied by changing the type of the perturbation. The present investigation deals primarily with triple charged OVs. The corresponding perturbations yield the most expedient results with respect to the channel separation and energy efficiency presently available by the authors.

Optical vortices are a special type of dark beams. They are characterized by their specific helical phase distribution in the transverse plane. At the phase singularity point in the screw-type phase distribution both the real and the imaginary parts of the field amplitude are zero and the intensity is zero too. Analytically the screw-type phase distribution is described by the function $\exp (\operatorname{im} \varphi)$, where $\varphi$ stands for the azimuthal coordinate and $m$ is an integer number called topological charge. In Fig. 8 the phase profile of an OVS is presented.

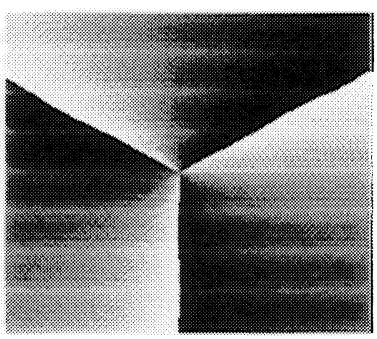

Fig. 8 Dark beam: Optical vortex (OV) with topological charge $m=3$ (phase profile). White and black denote phases of 0 and $2 \pi$, respectively.

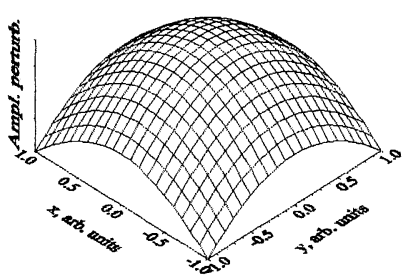

Fig. 9 Spiral-type amplitude a and phase $\phi$ of the perturbation of the guiding triple-charged OV beam:

$\mathrm{a}(\mathrm{z}=0)=\mathrm{a}_{0} \exp (\mathrm{im} \varphi) \exp \{\mathrm{i}(\mathrm{Kr}-\mathrm{N} \varphi)\} ; \phi(\mathrm{z}=0)=\phi_{0} \exp \{\mathrm{i}(\mathrm{Kr}-\mathrm{N} \varphi)\}$

The perturbation applied to the guiding OVS determines its splitting. In this work spiral type of perturbation is discussed: the field amplitude is given by $a(\mathrm{z}=0)=\mathrm{a}_{0} \exp (\mathrm{i} m \varphi) \exp \{i(K r-N \varphi)\}$ and the one concerning field phase distribution is described by the expression $\phi(z=0)=\phi_{0} \exp \{i(K r-N \varphi)\}$. Here $K$ and the integer $N$ stand for the radial and the azimuthal wave numbers of the perturbation. The profiles of both the amplitude and phase parts are presented in Fig. 9 in the case of $K=1$ and $N=3$. The probe beam is assumed to be Gaussian-shaped. The results of the numerical simulation are illustrated in Figs. 10 and 11. 

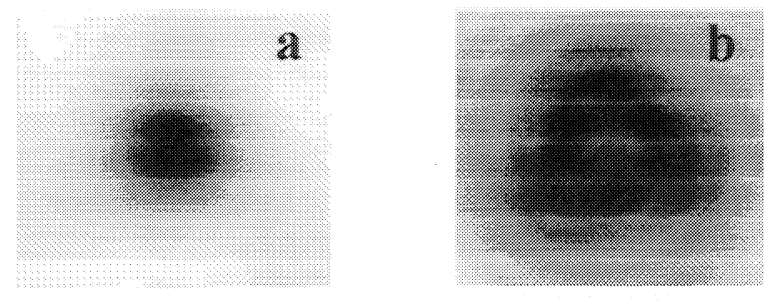

Fig. 10 Control beam input $(\zeta=0)$ and output $(\zeta=12)$ cross-section. (Scale factor $\mathrm{x} 2$ )
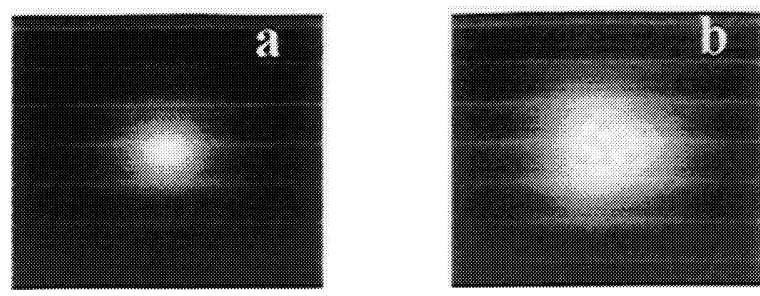

Fig. 11 Signal beam input $(\zeta=0)$ and output $(\zeta=12)$ cross-section.

As it could be seen by the figures the initial single beam is split into three sub-beams, thus producing an effective optical brancher. Two types of output channel distribution schemes are analyzed (Fig. 12). For both of the schemes the respective energy efficiencies for each of the channels are estimated. In scheme a) the initial energy is evenly redirected (17.6\% per channel). In scheme b) $13.7 \%$ of the initial energy is coupled to the central channel (4), and $20.0 \%$ - per each of the other three ones $(1,2$ and 3$)$.

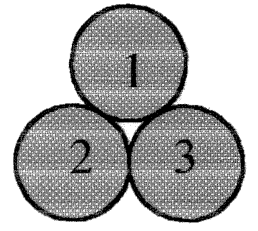

a)

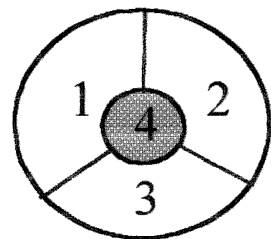

b)

Fig. 12 Output channel distribution schemes for branching by decaying of optical vortices

At this point it is difficult to say which of the schemes proposed is more efficient and advantageous. Further investigations on similar branching schemes based on decaying of multiple-charged OV beams are under way.

\section{CONCLUSION}

The analysis and the numerical simulation of dark beam propagation in non-linear medium show that the transverse deflection is significantly affected by the type of phase dislocation nested in. On the other hand because of the topological instability, through applying a proper kind of perturbation, a multiple-charged OV can be controllably split. These properties of the dark beams evoke the idea of producing an all-optical coupling and branching schemes which are promising for future practical applications. Possible coupling and branching schemes are proposed in the present work.

\section{ACKNOWLEDGMENTS}

This work was supported by the Science Foundation of the Sofia University (Sofia, Bulgaria).

\section{REFERENCES}

1. G. P. Agraval, Nonlinear fiber optics, (Academic, Orlando, 1989).

2. Yu. Kivshar, and Bary Luther-Davies, "Dark optical solitons: physics and applications," Physics Reports 298, pp. 81-197, 1998.

3. A. Dreischuh, G. G. Paulus, and F. Zacher, "Quasi-two-dimensional dark spatial solitons and generation of mixed phase dislocations," Appl. Phys. B69, pp. 113-117, 1999.

4. A. Dreischuh, G. G. Paulus, F. Zacher, and I. Velchev, "Steering one-dimensional odd dark beams of finite length," Appl. Phys., B69, pp.113-117, 1999.5

5 D. Neshev, A. Dreischuh, S. Dinev, and L. Windholz, "Controllable branching of optical beams by quasi-twodimensional dark spatial solitons," J. Opt. Soc. Am. B14, pp. 2869-2876, 1997.

6. D. Neshev, A. Dreischuh, S. Dinev, and L. Windholz, "Controllable branching of optical beams by quasi-twodimensional dark spatial solitons," Proc. SPIE, 3052, pp. 212-217, 1996.

7. I. Velchev, A. Dreischuh, D. Neshev, and S. Dinev, "Multiple-charged optical vortex solitons in bulk Kerr media," Opt. Commmun. 140, pp. 77-82, 1997.

8 A. Dreischuh, G. G. Paulus, F. Zacher, F. Grasbon, D. Neshev, and H. Walther, "Modulational instability ofmultiple- charged optical vortex solitons under saturation of the nonlinearity," Phys. Rev. E60,

pp.7518-7524, 1999. 